\title{
Assistência farmacêutica em casos de polifarmácia entre a população idosa
}

\author{
Pharmaceutical assistance in cases of polifarmacy between the elderly population
}

Asistencia farmacéutica en casos de polifarmacia entre la población idosa

\begin{abstract}
Anne Caroline Araújo Silva ${ }^{1}$, Bruno Oliveira Silva da Cruz ${ }^{1 *}$, Ernando Moreira da Costa ${ }^{1}$, Felipe da Silva Carvalho ${ }^{1}$, Francisco Honeidy Carvalho Azevedo ${ }^{1}$, Igor Alves dos Santos ${ }^{1}$, Maria Michelle Farias Silva ${ }^{1}$, Nágila Silva Alves ${ }^{1}$, Lucas Kevin Souza de Matos ${ }^{1}$, Vinicius José Campelo Duarte ${ }^{1}$, Viviane Leal Veloso ${ }^{1}$, Sabrina Sérgio Sousa Santos ${ }^{1}$.
\end{abstract}

\section{RESUMO}

Objetivo: O presente estudo teve como objetivo discutir as possíveis contribuições da assistência farmacêutica para o paciente idoso polimedicado. Método: Trata-se de um estudo bibliográfico de literatura na modalidade revisão integrativa. O levantamento de dados foi realizado pela internet (bancos de dados online), através de consultas em trabalhos científicos publicados (indexados) na Scientific Electronic Library Online - SciELO. A coleta de dados foi realizada a partir de um formulário e a obtenção dos dados foi observada qualitativamente, por meio de questionário. Resultados: A alta prevalência da polifarmácia e do uso de medicamentos potencialmente inapropriados entre os idosos. Variáveis como morar sozinho, baixa escolaridade, estado civil, sexo, idade, presença de doenças crônicas interferem negativamente na qualidade de vida do idoso. Considerações finais: A assistência farmacêutica é uma alternativa eficaz e de suma importância na obtenção da melhoria da qualidade de vida do idoso, reduzindo impactos da morbimortalidade relacionada ao uso de vários medicamentos.

Palavras-chave: Polifarmácia em idosos, Envelhecimento, Medicamento Potencialmente Inapropriados, Assistência farmacêutica.

\begin{abstract}
Objective: The objective of this study was to discuss the possible contributions of pharmaceutical care to polymededicated elderly patients. Method: This is a bibliographic integrative literature review. Data collection was carried out through the Internet (online databases), through consultations in published scientific works (indexed) in Scientific Electronic Library Online - SCIELO. The data collection was conducted using a form and the data collection was qualitatively observed through a questionnaire. Results: The high prevalence of polypharmacy and the use of potentially inappropriate drugs among the elderly. Variables such as living alone, low level of education, marital status, sex, age, presence of chronic diseases interfere negatively in the quality of life of the elderly. Final considerations: Pharmaceutical care is an effective and extremely important alternative in improving the quality of life of the elderly, reducing the impacts of morbimortality related to the use of several drugs.
\end{abstract}

Key words: Polypharmacy in the elderly, Aging, Potentially Inappropriate Medication, Pharmaceutical Assistance.

${ }^{1}$ Centro Universitário Santo Agostinho, Teresina-Piauí. *E-mail: brunooliveira210@hotmail.com

SUBMETIDO EM: 5/2019

ACEITO EM: 6/2019

PUBLICADO EM: 7/2019

REAS/EJCH |Vol. Sup. 28 | e999 | DOI: https://doi.org/10.25248/reas.e999.2019 Página 1 de 6 


\section{RESUMEN}

Objetivo: El presente estudio tuvo como objetivo discutir las posibles contribuciones de la asistencia farmacéutica para el paciente anciano polimedicado. Método: Se trata de un estudio bibliográfico de literatura en la modalidad de revisión integrativa. La recolección de datos fue realizada por internet (bases de datos online), a través de consultas en trabajos científicos publicados (indexados) en la ScientificElectronic Library Online - SCIELO. La recolección de datos se realizó a partir de un formulario y la obtención de los datos fue observada cualitativamente por medio de un cuestionario. Resultados: La alta prevalencia de la polifarmacia y el uso de medicamentos potencialmente inapropiados entre los ancianos. Las variables como vivir solo, baja escolaridad, estado civil, sexo, edad, presencia de enfermedades crónicas interfieren negativamente en la calidad de vida del anciano. Consideraciones finales: La asistencia farmacéutica es una alternativa eficaz y de suma importancia en la obtención de la mejora de la calidad de vida del anciano, reduciendo impactos de la morbimortalidad relacionada al uso de varios medicamentos.

Palabras clave: Polifarmacia en ancianos, Envejecimiento, Medicamento Potencialmente Inapropiado, Asistencia farmacéutica.

\section{INTRODUÇÃO}

No Brasil, a população idosa vem crescendo rapidamente, as projeções indicam que em 2050 a população brasileira será de 253 milhões de habitantes e com isso o acrescimo do número de mortes, isso devido a dominância de doenças crônicas, que tem passado a ocupar um lugar de destaque, de modo que os pacientes necessitem fazer uso de uma quantidade significativa de medicamentos. Sendo assim, o múltiplo uso de medicamento no paciente idoso pode ocasionar riscos à sua saúde, isso porque eles são mais sensíveis as reações adversas e às interações medicamentosas, devido a alterações orgânicas produzidas pelo avanço da idade (ASSIS DL, et al. 2016).

No processo natural de envelhecimento, ocorrem alterações fisiológicas no idoso, como a redução de mecanismos homeostáticos (processo que visa manter as condições do meio interno constante e independentes das variações do meio externo), aumento do tecido adiposo, redução das atividades metabólicas, alterando a farmacocinética dos medicamentos em níveis de absorção e biodisponibilidade. Esses dentre outros fatores direcionam o paciente à facilidade de adesão a polifarmácia, vendo no medicamento a solução para equilibrar as deficiências funcionais referido (CARVALHO ATF, et al. 2015).

Segundo Nascimento RCRM et al. (2017); é crescente o uso da polifarmácia na população brasileira, onde a mesma é definida como a utilização de vários medicamentos, que se constitui uma pratica clinica frequente, principalmente entre a população idosa. A polifarmácia dependendo do grau de agressividade da doença poderá constituir como uma das formas de tratamento necessário. Diante desse cenário, Carvalho ATF et al. (2015) diz que se faz importante o acompanhamento sistemático aos pacientes idosos que utilizam múltiplos medicamentos.

A assistência farmacêutica (AF) como uma estratégia de atenção à saúde é um mediador de informações adequadas para o uso correto do medicamento, além de ser uma ferramenta que orienta e reeduca o paciente (SILVA AM, et al. 2014). A Política Nacional de Medicamentos definiu assistência farmacêutica como um grupo de atividades relacionadas com o medicamento, destinadas a apoiar as ações de saúde demandadas por uma comunidade. Abrange o abastecimento de medicamentos em todas e em cada uma de suas etapas essenciais, a conservação e controle de qualidade, a segurança e a eficácia terapêutica dos medicamentos, o acompanhamento e a avaliação da utilização, a obtenção e a difusão de informação sobre medicamentos e a educação permanente dos profissionais de saúde e da população (MARQUES AEF, et al. 2017).

O Farmacêutico pode atuar no melhoramento da saúde do idoso, nas medidas de AF, na qual o profissional poderá agir prestando informações quanto a doença e ao tratamento assim como prestar a atenção farmacêutica, além de contribuir para que os pacientes recebam uma terapia medicamentosa segura e eficaz.

REAS/EJCH |Vol. Sup. 28 | e999 | DOI: https://doi.org/10.25248/reas.e999.2019 Página 2 de $\mathbf{6}$ 
O aconselhamento aos doentes acerca do uso de medicamentos de venda livre, a participação em programas de educação para a saúde em colaboração com outros membros da equipe de saúde são algumas estratégias de como a AF pode ajudar a população idosa (MARQUES AEF, et al. 2017). Dessa forma o estudo tem como objetivo discutir as possíveis contribuições da AF para o paciente idoso polimedicado.

\section{METODOLOGIA}

Trata-se de um estudo bibliográfico de literatura na modalidade revisão integrativa. Onde os estudos de revisão têm como principal objetivo buscar conceitos, revisar teorias e evidências sobre um tema específico e a partir dele, possam ser discutidas as possíveis contribuições da AF para o paciente idoso polimedicado (SOUZA MT, et al. 2010).

O levantamento de dados foi realizado pela internet (bancos de dados online), entre os meses de fevereiro e março de 2019, através de consultas em trabalhos científicos publicados (indexados) na Scientific Electronic Library Online - SciELO, Literatura Latino-Americana e do Caribe em Ciências da Saúde (LILACS); utilizouse as seguintes combinações descritores: AF para idosos, polifarmacia em idosos e fatores associados à polifarmácia em idosos.

Utilizou-se como critério de inclusão para a seleção de amostras os artigos indexados de 2014 a 2019, em periódicos nacionais e internacionais, disponibilizados na íntegra em língua portuguesa, inglesa e espanhola e que respondam a temática do estudo.

Foram excluídos da população pesquisada, publicações cujos títulos e/ou objetivos não possuam ligação direta com os descritores supracitados, não respondam a temática ou fujam ao objeto de estudo, apresentem resultados ambíguos ou inconclusivos. A coleta dos dados foi executada a partir de um formulário e a obtenção dos dados foram observados qualitativamente, por meio de questionário.

\section{RESULTADOS E DISCUSSÃO}

Os artigos pesquisados foram um total de 40 , onde foram excluídos dentre estes 25 , pois não estavam relacionados diretamente ao tema em questão, sem relação com os objetivos do trabalho, os que se repetiram nas pesquisas listadas e os que foram publicados anteriores ao ano de 2013. A amostra final desta revisão foi constituída por 15 artigos científicos, selecionados pelos critérios de inclusão previamente estabelecidos.

\section{Medicamentos potencialmente inapropriados para idosos}

Segundo Gorzoni ML et al. (2012); o Critério de Beers foi criado em 1991 com o objetivo de listar os medicamentos potencialmente inadequados para idosos. Medicamentos são potencialmente inapropriados (MPI) quando o risco de ocasionar efeitos colaterais é superior aos benefícios em idosos.

As próprias alterações fisiológicas que surgem no organismo com o envelhecimento ajudam no aparecimento de tais efeitos. A frequência de comorbidade (presença de duas ou mais doenças em concomitância na mesma pessoa) também tende a potencializar a utilização de MPI.

Segundo Lutz BH et al. (2017); com o envelhecimento da população, a morbidade e mortalidade ganham maior visibilidade nos cuidados à saúde, já que essas condições estão associadas com o mesmo, e consequentemente, o idoso apresentará delimitações funcionais que demandarão cuidados constantes. $O$ metabolismo hepático, os mecanismos homeostáticos e a função renal podem ficar comprometidos devido a farmacologia do idoso. Disso dá-se a dificuldade de eliminação de metabólitos, a concentração de substâncias tóxicas e os possíveis efeitos colaterais.

No seu estudo os Medicamentos Potencialmente Inapropriados (MPI) continuam sendo prescritos como tratamento de primeira linha para esses pacientes. As mulheres são mais vulneráveis e se encontram com maior predomínio na utilização de MPIs, já que são mais dispostas a procurar auxílio médico e se expressar sobre problemas de saúde (LUTZ BH, et al. 2017). 
Lopes LM et al. (2016); acrescenta ainda que a compreensão e a propagação de informações sobre os MPI é uma ferramenta importante de suporte na melhoria da qualidade da assistência ao paciente idoso, visto que o uso adequado de medicamentos por essa faixa etária é fundamental para a prevenção de efeitos colaterais, que comprometem a saúde e a qualidade de vida deles.

Helena ETS et al. (2015); afirmam que compreender como os usuários apreciam o acesso aos medicamentos é fundamental para que se possa refletir as práticas profissionais e interferir sobre a forma de organização dos serviços, focando em sua melhoria. Complementa ainda que, é necessário um elo de confiança entre aquele que entrega o medicamento e o usuário, facilitando a comunicação, já que a comunicação é peça chave para melhoria das práticas assistenciais. E conclui que a promoção do entendimento do usuário sobre o medicamento que lhe é prescrito influencia positivamente no seu tratamento.

Pagno AR et al. (2018); realizou uma pesquisa populacional, onde nos seus resultados mostravam a prevalência do uso de medicamentos inapropriados por idosos e ainda identificou que $39,4 \%$ daquelas pessoas usavam vários medicamentos, ou seja, a polifarmácia. Pesquisa realizada também por Marques EFM et al., 2018, onde demostra que a maioria das pessoas que fazem uso de polifarmácia ou uso de medicamento potencialmente inapropriados é do sexo feminino.

A Assistência Farmacêutica (AF) é de suma importância para a população, principalmente os idosos, pois são os que mais utilizam medicamentos e consequentemente são os mais propícios à automedicação ou uso de polifarmácia podendo causar interações indesejadas. O farmacêutico é amplamente conhecedor dos medicamentos com conhecimentos técnicos superiores a qualquer outro profissional. Assim, o farmacêutico é capacitado para intervir com qualquer irregularidade medicamentosa, aconselhando os pacientes ou até mesmo intervir receitas, através de processos bem documentados, para evitar erros de prescrições e dosagem de medicamentos (FARIAS FR, et al. 2018).

Uma pesquisa feita por Tavares DS et al. (2018); evidencia alta prevalência do uso de polifarmácia entre os idosos, que pode ser caracterizado por vários fatores como doenças crônicas, dificuldade de acesso ao posto de saúde/hospital, questão financeira, automedicação, escolaridade dentre vários outros fatores. Com esses achados e sabedor de que os idosos são mais frágeis as susceptíveis situações, também aumentam as possíveis interações medicamentosas. Marques GFM et al., 2018 alega que o farmacêutico deve intervir por meio da consulta farmacêutica nas prescrições com MPI e polifarmácia.

Segundo Rocha CH et al. (2008); no que se refere ao aspecto da orientação, o profissional farmacêutico pode contribuir explicando aos pacientes sobre sua patologia e os medicamentos a serem administrados. A orientação, inclui instruções sobre o modo correto de usar, possíveis efeitos adversos, atitude a tomar no caso de esquecimento de doses, alertas quanto aos riscos da automedicação e da descontinuação prévia do tratamento. Caso o farmacêutico detecte algum problema com a terapia farmacológica prescrita, ou mesmo de dificuldade de adesão, deve entrar em contato com o médico para discutirem uma possível intervenção.

Martins GA et al. (2015); observou que há uma alta prevalência do uso de MPI e o envelhecimento está diretamente proporcional ao seu uso como também a frequência de doenças cônicas. Como consequência disso, o idoso fica exposto à polifarmácia, posteriormente sujeito ao surgimento de novos problemas, agora relacionados aos efeitos adversos dos medicamentos.

Segundo Nascimento RCRM et al. (2017); para a melhoria da adesão à terapia medicamentosa e, em consequência, os resultados clínicos, se faz necessário os serviços de acompanhamento farmacêutico, com consultas para elaborar esquemas de cuidado, resolver os impasses relacionados aos medicamentos e oferecer acompanhamento congruente, com foco na aquisição de habilidades e competências para a cor responsabilização.

A avaliação regular dos recursos terapêuticos, com foco na adesão, adaptação às preferências individuais e reconhecimento de riscos pode reduzir danos e potencializar os benefícios almejados. Uma pesquisa realizada por Cassoni TCJ et al., 2014 apontou que os idosos que utilizam MPI possuem maior risco de mortalidade, independente dos fatores socioeconômicos ou uso de vários medicamentos. Isso está 
comumente ligado a prática da automedicação e idade. Geralmente os idosos possuem saúde mais instáveis, e muito das vezes, por razões pessoais ou até mesmo por dificuldade de se locomoverem, eles preferem obter pelo método mais "fácil" e prático, a automedicação.

\section{Fatores associados a polifarmácia}

A polifarmácia já é evidente, definida como a utilização de cinco ou mais medicamentos, ou utilização de mais medicamentos do que está clinicamente indicado. Essa situação ocorre com maior frequência em indivíduos com doenças crônicas e por conta do envelhecimento. Ainda existem outros fatores que influenciam como, por exemplo, morar sozinho e ser do sexo feminino, baixa escolaridade, ter mais de quatro doenças, plano privado de saúde são fatores relacionados a polifarmácia. Os medicamentos simbolizam o principal agente tóxico, sendo que a automedicação, uso indevido de antibióticos e medicamentos injetáveis estão relacionadas à polifarmácia (SALES AS, et al. 2017).

Conforme Almeida NA et al. (2017); um elevado consumo de medicamentos afeta negativamente a qualidade de vida do idoso, porem esses mesmos medicamentos são os que ajudam a prorrogar a vida, deste modo a polifarmácia não é necessariamente à que apresenta potenciais riscos para eventos adversos, mais sim o uso indevido. Como os idosos apresentam numerosas comorbidades, as prescrições de medicamentos devem ser revisadas frequentemente, desta maneira, a presença do farmacêutico dando suporte e assistência ao paciente idoso é de suma importância para a promoção do uso racional de medicamentos, diminuindo os erros de prescrições, além de prevenir o uso incorreto.

Assim como Farias FR et al. (2018); relatou sobre a importância da Assistência Farmacêutica (AF), Sachy M, 2018 também cita em sua pesquisa que o profissional farmacêutico agrega grandes valores aos serviços de saúde, devido à implantação mais incisiva da AF nos polos de saúde, ajudando a promover melhorias na saúde para os doentes e aqueles que carecem de cuidados mais específicos. De fato, a AF deve ser bem vista pela população e bem mais usufruída pelo mesmo, pois o farmacêutico tem totais condições de aplicar os cuidados necessários ao paciente com dificuldades em suas medicações, seja na posologia, dosagem ou até mesmo como usar corretamente.

Segundo o Conselho Federal de Farmácia (CFF), a atenção farmacêutica é determinada por ações do farmacêutico, cujo o paciente é o principal beneficiário. Desta forma, o farmacêutico atua de fato na assistência ao paciente, responsabilizando-se, junto da equipe multiprofissional, pela segurança e pela eficácia da farmacoterapia. Isto se dá por meio da identificação, da resolução e da prevenção dos problemas relacionados a medicamentos (PRM). A intervenção farmacêutica (IF), com a constante monitorização farmacoterapêutica, é capaz de reduzir PRM, aumentar a eficácia e reduzir os riscos da farmacoterapia. (DOBLINSKI DL, et al. 2006).

Em uma pesquisa realizada por Farias FR et al. (2018); discutindo sobre os serviços farmacêuticos, ele concluiu que os serviços prestados pelo farmacêutico, como por exemplo, AF dentre vários outros serviços, contribui muito para o controle de doenças entre a população. Consequentemente diminui a periodicidade de pessoas nos postos de saúde e leito de hospitais. E ainda cabe a AF repassar à população quanto ao uso racional do medicamento, educando as pessoas a terem um melhor proveito com 0 uso de seus medicamentos como também tirando qualquer dúvida sobre o seu uso. Afinal, é o farmacêutico o profissional habilitado à dispensação e educação medicamentosa.

\section{CONSIDERAÇÕES FINAIS}

Em síntese, verificou-se elevado consumo de medicamentos pelos idosos na pesquisa bem como, o uso de polifarmácia e de MPI. Isso pode ser caracterizado por vários fatores, como doenças crônicas, baixa escolaridade, automedicação, e idosos que não moravam acompanhados. Idosos que mais utilizam MPIs possuem maior risco de mortalidade, independente dos fatores socioeconômicos e do uso da polifarmácia, resultados que evidenciam que alguns aspectos sociais e de condição de saúde exercem importante papel no uso de múltiplos medicamentos entre os idosos. Os erros mais comuns da utilização de medicamentos 
entre os idosos envolvem medicamentos impróprios, dosagem errada e combinações impróprias dos medicamentos. Portanto conclui-se que a AF é uma alternativa eficaz e de suma importância na obtenção da melhoria da qualidade de vida do idoso, reduzindo impactos da morbimortalidade relacionada ao uso de vários medicamentos. O farmacêutico é corresponsável no monitoramento dos resultados terapêuticos e efeitos adversos, sendo de suma importância para o acompanhamento de idosos em uso de polifarmácia.

\section{REFERÊNCIAS}

1. ALMEIDA NA, et al. Prevalência e fatores associados à polifarmácia entre os idosos residentes na comunidade. Rev. bras. geriatr. gerontol. vol.20 no.1 Rio de Janeiro Jan./Feb. 2017

2. ASSIS DL, et al. Polifarmacia e uso de medicamentos inapropriados em idosos institucionalizados: lições ainda não aprendidas. Goiânia v.10, n. 3 p.126-31, 2016.

3. CARVALHO ATF, et al. Polifarmacia e automedicação em idosos. II congresso brasileiro de ciências da saúde. v.30, n. 1, p. 1-6, 2015.

4. CASSONI TCJ, et al. Uso de medicamentos potencialmente inapropriados por idosos do Município de São Paulo, Brasil: Estudo SABE. Cad. Saúde Pública, Rio de Janeiro, 30(8):1708-1720, ago, 2014.

5. DOBLINSKI PMF, et al. Assistência e atenção farmacêutica: estudo comparativo entre dois bairros de classes sociais diferentes em toledo-pr. Infarma, v.18, no 9/10, 2006

6. FARIAS FR, et al. Avaliação dos serviços farmacêuticos na Atenção Primária à Saúde no cuidado ao paciente com tuberculose. Saúde debate vol.42 no. spe2 Rio de Janeiro Oct. 2018.

7. HELENA ETS, et al. Percepção dos usuários sobre acesso aos medicamentos na atenção primária. Cad. Saúde colet., Rio de Janeiro, v. 23, n. 3, p. 280-288, set. 2015.

8. LOPES LM, et al. Utilização de medicamentos potencialmente inapropriados por idosos em domicílio. Ciênc. saúde coletiva [online]. 2016, vol.21, n.11, pp.3429-3438. ISSN 1413-8123.

9. LUTZ BH, et al. Inadequação do uso de medicamentos entre idosos em Pelotas, RS. RevSaude Publica. 2017; 51:52.

10. MARQUES AEF, et al. Assistência farmacêutica: uma reflexão sobre o papel do farmacêutico na saúde do paciente idoso no brasil. Temas em saúde. Joao Pessoa, v. 17, n. 3, p.129-146, 2017.

11. MARQUES GFM, et al. Polifarmácia e medicamentos potencialmente inapropriados para idosos na enfermagem gerontológica. Revista Brasileira de Enfermagem, 2018.

12. MARTINS GA, et al. Uso de medicamentos potencialmente inadequados entre idosos do Município de Viçosa, Minas Gerais, Brasil: um inquérito de base populacional. Cad. Saúde Pública [online]. 2015.

13. MINISTÉRIO DA SAÚDE; CONSELHO NACIONAL DE SAÚDE. Resolução no 338, de 06 de maio de 2004.

14. NASCIMENTO RCRM, et al. Polifarmácia: uma realidade na atenção primária do Sistema Único de Saúde. RevSaude Publica, Belo Horizonte, v. 3, n.2, 2017.

15. PAGNO AR, et al. A terapêutica medicamentosa, interações potenciais e iatrogenia como fatores relacionados à fragilidade em idosos. Rio de Janeiro, 2018.

16. ROCHA CH, et al. Adesão à prescrição médica em idosos de Porto Alegre, RS. Ciênc. Saúde coletiva, Rio de Janeiro, v. 13, supl. p. 703-710, abr. 2008

17. SACHY M, et al. Assistência Farmacêutica em Moçambique: a ajuda externa na provisão pública de medicamentos. Ciênc. Saúde coletiva vol.23 no.7 Rio de Janeiro July 2018.

18. SALES AS, et al. Perfil farmacoterapêutico e fatores associados à polifarmácia entre idosos de Aiquara, Bahia, em 2014. Epidemiol. Serv. Saúde [online]. 2017.

19. SILVA AM, et al. Atenção farmacêutica ao idoso no uso da polifarmácia. São Paulo, v.2, n.3, 2014.

20. SILVA PLN, et al. Atenção farmacêutica e os potenciais riscos da polifarmácia em idosos usuários de uma farmáciaescola de Minas Gerais: aspectos socioeconômicos, clínico e terapêutico. J Health BiolSci, p. 247-252, 2017.

21. TAVARES DS, et al. Perfil de idosos com síndrome metabólica e fatores associados às possíveis interações medicamentosas. Rev. Bras. Geriatr. Gerontol, Rio de Janeiro, 2018; 21(2): 168-179. 